Gynäkol Geburtshilfliche Rundsch 1995;35:243

\title{
Autorenregister Vol. 35, 1995
}

Das Supplement 1ein eigenes Autorenregister

Abels, C. 90 Albrecht, A.E. 51 A $1 / 8$ e,A. 118 Arras, H. 98

Backe,J. 79,199 Bajardi,F. 188 Bajka,M. 38 Balks, HJ. 215 Baumgarten, K. 183 Baumgartner, R. 90 Bender, H.G. 169 Berg,D. 64 Beyer, W. 90 Bösch,H. 114 Breitenecker, G. 16

Brezinka,C. 58,229,238 Buddeberg,C. 20 Buhner, M. 98

Chiari,A. 173 Concin,H. 114

Dapunt, O. 122 Deix,R. 116 Dellian,M. 90 Dennemark 112 Desoye, G. 12 Dietl,J. 112

Döinghaus, K. 98 Dresel,V. 98

Enzelsberger, H. 173 Eppel,W. 159

Fink, D. 26, 29 Fischl, F. 54 Frigo,P. 159

Gaulrapp,K. 199 Geringer-Volgger, B. 118

Gitsch,E. 164 Gitsch,G. 16,42 Goetz,A.E. 90 Gosch,M. 173 Günter,H.-H. 215

Haller,U. 1,20,26,38,63,13 Hammerstein, J. 49 Hanzal,E. 173 Hartmann, B.W. 51 Häusler,G. 173 Häusler, M. 234 Helmer,H. 173 Hepp,H. 1,63,72,85, 131 Hickl,E. 112 Höckel,M. 133 Hohmann, M. 112 Horn,U. 98 Huber,J.C. 159

Kaesemann, H. 79 Kainz, Ch. 16 Kimmig, R. 72 Kirschstein, M. 85,90 Kiss, G. 221 Köchli, O.R. 26, 29, 38 Kohlberger, P. 16 Kölbl,H. 16, 123,139, 173 Koletzko,B. 2 Kölle,D. 221 Korell,M. 85,90 Krause,B.Th. 194 Kries, R. von 2 Kubista,E. 126 Kucera,E. 209 Kucera, H. 209 Kunz,J. 34 Künzel,W. 112

Lang,C. 159 Lang, N. 98

Lauermann, E. 159 Leodolter, S. 142 Lindenmann, J. 46

Madersbacher, H. 221 Mähr,R. 34 Mair,D. 221 Mamsen, A. 179 Marth,C. 118,122 Martius, J. 79 Möller,S. 194 Möse,J.R. 234

Niesert, St. 215 Nitschmann, A. 215

Ott,M. 79

Penz-Koza,A. 118 Perucchini, D. 26, 29 Pieber,D. 170,175

Rabe,Th. 49 Ralph, G. 175 Rath,W. 112 Reichel,R. 56 Reinold,E. 1,63,131,132,

159,178,180,240 Reinthaller, A. 16, 123 Rempen, A. 226 Renziehausen, K. 112 Robbiani, M. 38 Roos, T. 79 Runnebaum, B. 49

Sam,C. 173 Schaller,A. 153,158 Schär,G. 26,29,184 Scheidel,P. 72 Schmidt, D. 38 Schmidt, W. 112

Schneider, K.T.M. 112 Schulz-Wendtland, R. 98 Schwärzler, P. 114 Seehaus, D. 72 Seifert,M. 126 Sevelda,P. 149 Slunsky,R. 6 Solder, E. 118 Staff, A. 179 Stallmach,T. 38 Steck,T. 199

Steiner,R.A. 20

Tamussino, K. 170,175 Tempfer, C. 16 Teschler-Nicola, M. 159 Tscherne, G. 177

Untch,M. 85,90 Urdl,W. 12

Vander-Möse, A. 234 Vavra,N. 209 Vogl,S. 126 Vondruska-Benkert, K. 20

Wedeking-Schöhl, H.H. 215 Weihs,E. 182 Weise,W. 112 Weitzel,H. 112 Wierrani, F. 182

Wilmanns, J.C. 103 Winter, R. 120,234 Wolff, F. 112 
Zahradnik, H.P. 112 Zivkovic,F. 170,175 